\title{
Three Cases of Aberrant Right Subclavian Artery in the Dog
}

In the normal dog only 2 arteries leave the ascending aorta - the brachiocephalic trunk and the left subclavian artery. This communication describes 3 dogs, a labrador, a poodle and a german shepherd, in which 3 large arteries left the ascending aorta - firstly a trunk for both common carotid arteries, then a left subclavian artery and finally a right subclavian artery.
The dogs had been donated to the Department of Anatomy for use in teaching. Immediately after death, they were perfused with approximately 31 of $4 \%$ formaldehyde through the left common carotid artery, followed by injection of some $300 \mathrm{ml}$ of red latex solution to facilitate dissection of arteries. After fixation the dogs were placed in a vat of $30 \%$ ethanol for 2 months to wash out the formalin.

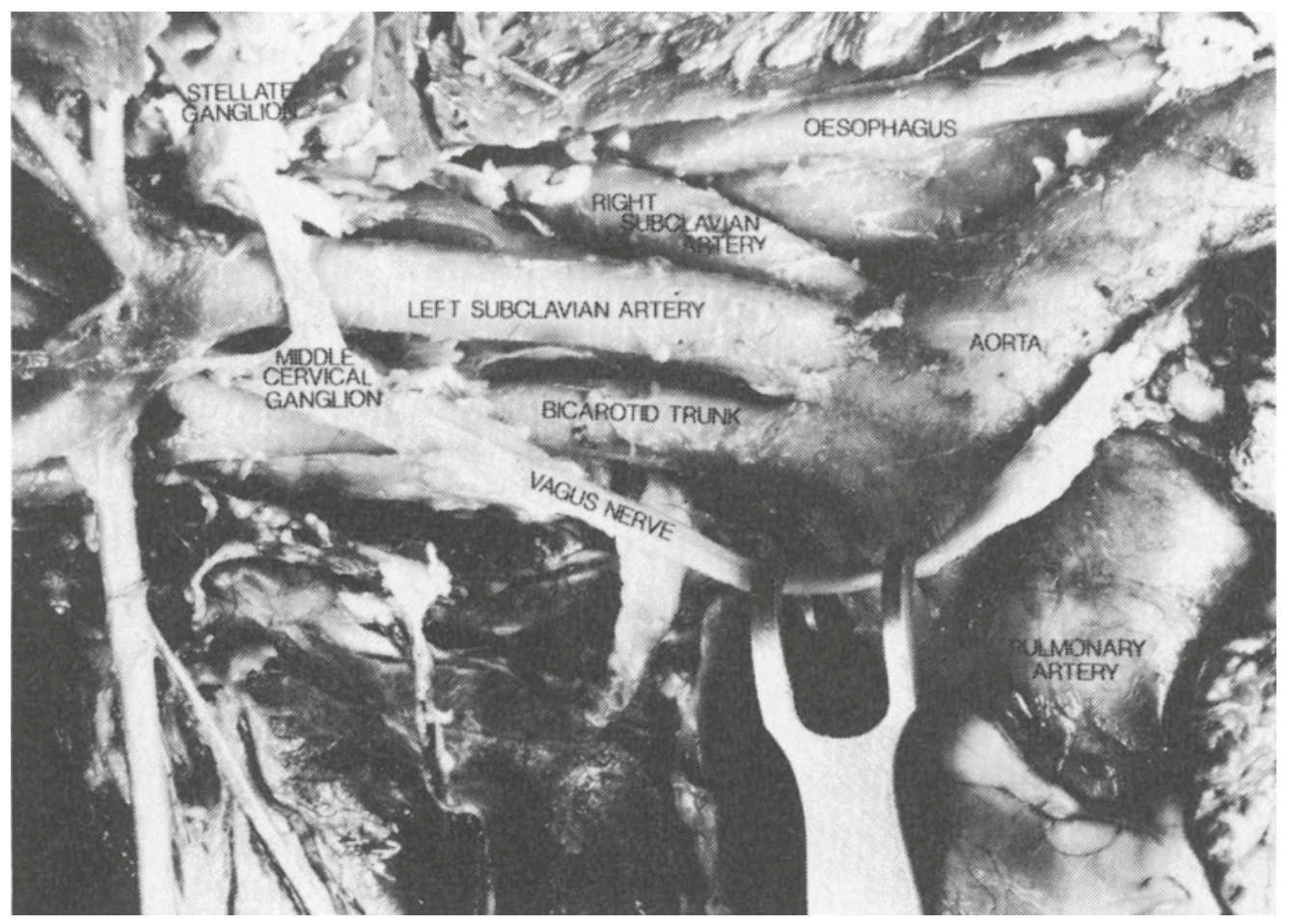

Fig. 1. Three large vessels emerge from the ascending aorta. The proximal (lowest) is the "bicarotid" trunk. The next is the normal left subclavian artery and the third, the origin of which is partly obscured, is the right subclavian artery. The vagus nerve, thoracic duct, ansa subclavia and stellate and middle cervical ganglia have been preserved to show their relationships to the vessels. 
The anatomy of the vasculature was identical in each dog (Fig. 1). The most proximal artery was a bicarotid trunk, which left the aorta at its most anterior point. It ran cranial along the left wall of the trachea to a point level with the ansa subclavia. Here it divided into left and right common carotid arteries, the left continuing directly forwards along the trachea and the right artery crossing under the trachea to the right side. Normally the most proximate artery to leave the aorta is the brachiocephalic trunk, which divides to give first the 2 carorid arteries and then the right subclavian artery.

The second artery was the left subclavian. It left the aorta to the left of its median plane with the dorsal edge of the trachea. Nor- mally the left subclavian artery leaves the aorta from its median plane but in this case it has been pushed to the left by the third artery. It then ran forwards to the stellate ganglion where it divided into its 5 terminal branches, the vertebral artery, the costocervical trunk, and the internal thoracic, superficial cervical and axillary arteries. Apart from its abnormal origin on the aorta, the anatomy of this artery seemed normal.

The third artery was a right subclavian, which left the aorta to the right of its median plane slightly above the left subclavian. Normally the right subclavian artery is a direct continuation of the brachiocephalic trunk. It coursed obliquely cranial and dorsal, then crossed over to the right side be-

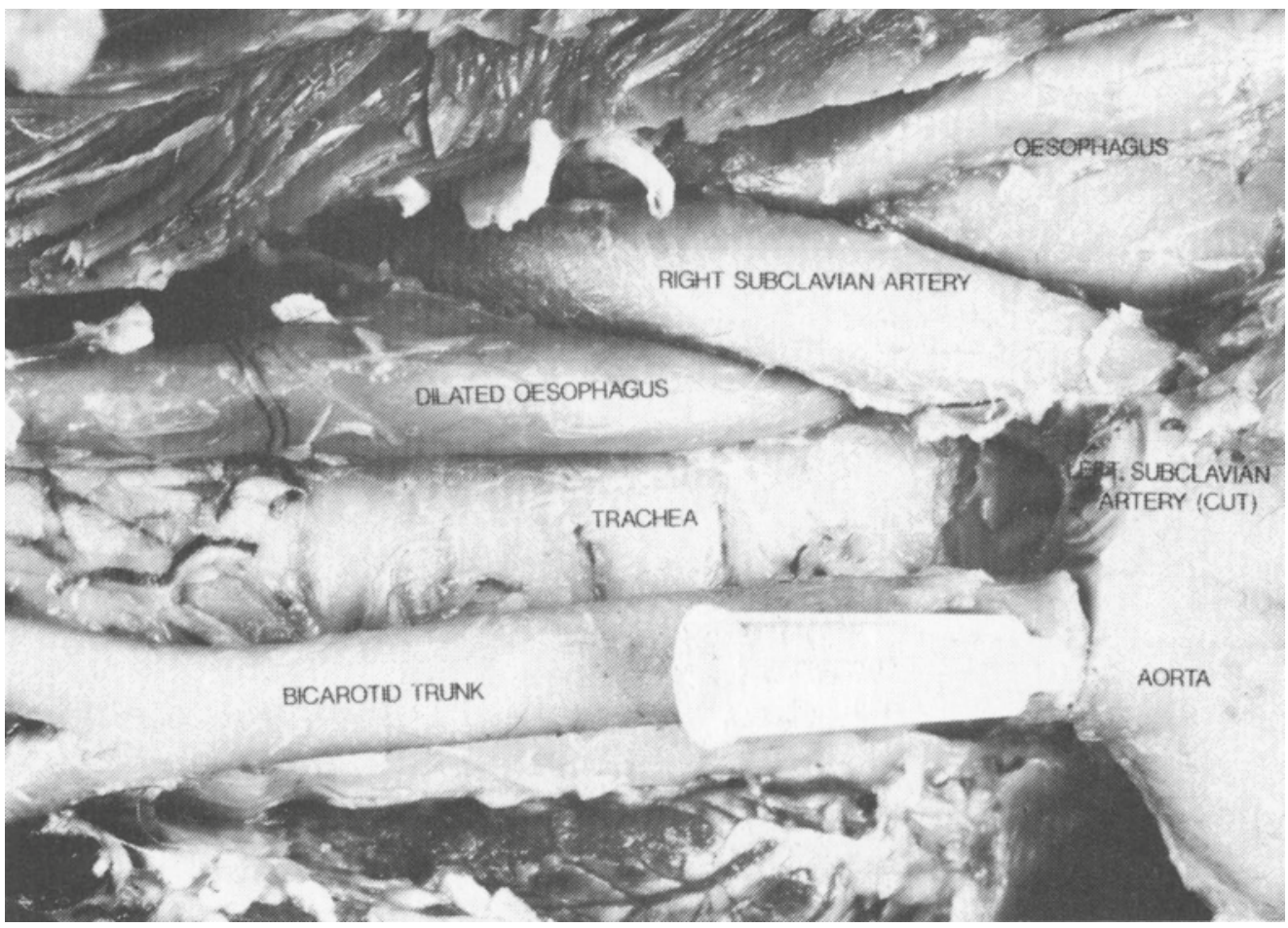

Fig. 2. The right subclavian artery, while on its way over to the right side, has compressed the oesophagus against the trachea. The left subclavian artery has been removed to reveal the trachea in better detail. The foreign body to the lower right is a needle holding the bicarotid trunk in place. 
tween the oesophagus and the dorsal chest wall (Fig. 2). In doing so it squeezed the oesophagus against the trachea, resulting in a moderate dilatation of the oesophagus on the dorsal border of the trachea. One of the dogs had in addition a dilatation of the caudal part of the oesophagus immediately cranial to the diaphragm but there is no obvious connection between this and the abnormal placement of blood vessels cranial to the heart.

These cases of right subclavian artery are noteworthy because of the constriction produced in the oesophagus. There was no prior history of regurgitation in the case of the single dog for which records exist and, judging by the other animals' good condition, it is doubtful that they had had problems either. We have now seen 3 such cases among the last 26 dogs donated to us which suggests that the condition may be more common than previously reported (Vitums 1962), at least in Norway. The other common vascular malformation which compromises the oesophagus is a persistent right aortic arch (PRAA), in which the oesophagus is squeezed between the right aorta, the ligamentum arteriosum and the base of the heart.

David Griffiths,

Department of Anatomy, Norwegian College of Veterinary Medicine.

\section{References}

Vitums A: Anomalous origin of the right subclavian and common carotid arteries in the dog. Cornell Vet. 1962, 76, 215-219.

(Received July 12, 1989).

Reprints may be requested from: David Griffiths, Department of Anatomy, Norwegian College of Veterinary Medicine, P. O. Box 8146, Dep. N-0033 Oslo 1, Norway. 
